\title{
Adherencia al tratamiento de los cuidadores de niños con Trastorno del Espectro Autista
}

\section{Fernanda de Melo Parente, Francisco Alcantud Marín, y Amparo Oliver Universidad de Valencia (España)}

El estudio está centrado en la adherencia al tratamiento de la familia del niño o niña con Trastorno del Espectro Autista (TEA) o sospecha de diagnóstico, identificando los elementos que justifican el proceso de adherencia. A partir de esa identificación, desarrollar una escala de medida para su estudio. Para ello se evaluó la adherencia con la aplicación de un cuestionario en un grupo de 128 padres de niños con TEA y con sospecha de diagnóstico, considerando como variables: los factores sociales, económicos, familiares e individuales. Los resultados muestran la importancia de la relación de esos factores con la adherencia en la adaptación al TEA tales como: el apoyo social, satisfacción con la vida y cohesión familiar.

Palabras-clave: Trastornos del Espectro Autista, adherencia al tratamiento, modelo de evaluación, intervención con la familia.

The study focuses on the adherence to treatment of the family of the child with Autism Spectrum Disorder (ASD) or suspected diagnosis, identifying the elements that justify the adherence process. From that identification, develop a scale of measurement for its study. For this, adherence was evaluated with the application of a survey in a group of 128 parents of children with ASD and with suspected diagnosis, considering as variables: social, economic, family and individual factors. The results show the importance of the relationship of these factors with adherence in the adaptation to ASD such as: social support, life satisfaction and family cohesion.

Keywords: Autism Spectrum Disorders, treatment adherence, evaluation model, family intervention.

Correspondencia Fernanda de Melo Parente. Calle Pianista Amparo Iturbi, $\mathrm{n}^{\circ}$ 35, 2. C.P.:46007. Valencia (España). E-mail: ferdeme@alumni.uv.es 
La expresión adherencia al tratamiento es definida por la Organización Mundial de la Salud como: "el cumplimiento del mismo; es decir, tomar la medicación de acuerdo con la dosificación del programa prescrito; y la persistencia, tomar la medicación a lo largo del tiempo" (OMS, 2003). Este concepto, según la OMS, está aplicado a la práctica médica, por referirse exclusivamente al uso de los medicamentos por parte del paciente. Partiendo de este limitado alcance, entendemos que la adherencia al tratamiento psicológico es un fenómeno más complejo por poseer un carácter multidimensional, dado que la adherencia no hace referencia a un sólo comportamiento sino a un conjunto de factores y/o variables (Martín y Grau, 2004). Es importante subrayar que la primera dificultad que nos encontramos está relacionada con la definición y diferenciación conceptual de la adherencia (adherence) y el cumplimiento (compliance) terapéutico (Fleta, Artiles, y Miragaia, 2009). En algunas publicaciones el término es empleado como sinónimo generando dificultades por la imprecisión del término (Nieto y Pelayo, 2009).

Entendemos que la adherencia psicológica está más allá de simplemente acudir a las citas y tomar las medicaciones, envuelve una variedad de comportamientos familiares, en ocasiones incluso puede comportar la modificación estructural tanto del ambiente como de las responsabilidades en el seno de la familia. La conducta adherente depende de otros factores como, por ejemplo, la relación familia-terapeuta y las características subjetivas individuales de cada miembro de la familia (Nieto y Pelayo, 2009). También, del impacto personal que el diagnóstico de TEA tenga en cada miembro de la familia, nivel cultural y educativo de los mismos, etc.

Es importante tener claro dos conceptos básicos para la comprensión de la adherencia. El primero, la asistencia al tratamiento que se define simplemente como la presentación del paciente durante una sesión programada y acordada con el terapeuta; y el segundo es el compromiso de un paciente en el tratamiento que se puede definir mediante la adherencia al tratamiento o la aceptación de las estrategias de tratamiento. La asistencia es necesaria para que el tratamiento sea cumplido y para que se logren los resultados, eso significa que la asistencia a la sesión por sí sola no describe efectivamente el compromiso con el tratamiento (Gopalan et al., 2010). El término compromiso, según McKay y Bannon (2004), abarca un proceso de varias fases que comienza con el reconocimiento de los problemas de salud mental del niño por parte de los padres, maestros u otros adultos dentro del contexto del niño y termina con un niño que recibe atención de salud mental. La familia busca atención en salud mental infantil para abordar y solucionar las necesidades y problemas no satisfechos entre el niño y la familia. Ese proceso se divide en dos pasos: uno la asistencia inicial y el otro, es la participación continua, los cuales se considera relacionados entre ellos mismos, pero cada uno dependerá de las características del niño, de la familia y del sistema de servicio sanitario (Figura 1). 

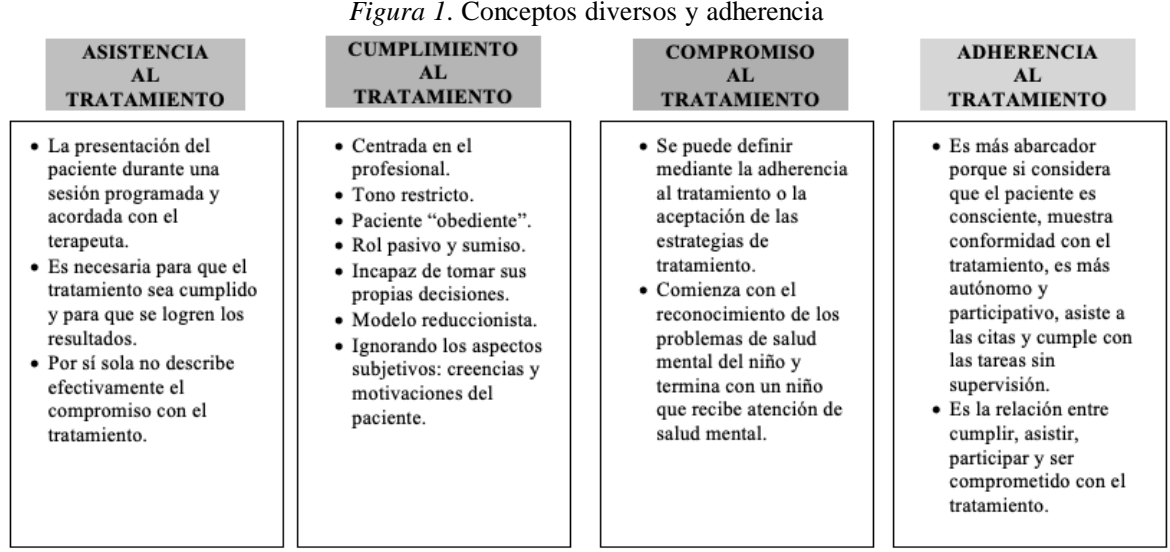

Elaboración propia a partir de integración de aportaciones teóricas de Martín y Grau (2004), Fleta et al. (2009), Gopalan et al. (2010) y McKay y Bannon (2004).

En los últimos años, las evidencias sobre los resultados de la intervención temprana en niños con TEA son abrumadoras (Barnett, 1987; Casto y Mastropieri, 1986; Guralnick y Bennett, 1987; Guralnick, 1998; McCormick, Brooks-Gum, Buka, Goldman, y Yu, 2006; Reynolds, Temple, Robertson, y Mann, 2001; Shonkoff y HauserCram, 1987; entre otras como se cita en Alcantud y Alonso, 2015). Sobre todo, con la participación activa de los cuidadores principales (padre/madre) en los programas de intervención (Guralnick, 2017). Pero ¿se ha estudiado la adherencia al tratamiento o, mejor dicho, se ha estudiado las causas de abandono del mismo? ¿Ha experimentado un progreso similar o significativo?

La familia es para el sujeto su primer núcleo de convivencia y de actuación; razón por la cual, son los primeros a darse cuenta de los problemas en el desarrollo del niño. Entonces, una de las funciones de la familia es que ella es la fuente de apoyo al individuo ante una dificultad y la principal responsable por la eficacia del tratamiento y lograr los cambios deseados. Así que, la presencia de un niño con TEA afecta a todos los miembros de la familia; en particular la persona quien desempeña el papel de cuidador, porque éste ve alterada su calidad de vida, sus actividades cotidianas, sus relaciones sociales, su salud y enfrenta una sobrecarga de trabajo, todo implicado a la atención del niño. En síntesis, la familia es la principal responsable por la adhesión en el tratamiento con el intuito de prevenir los episodios de agravamiento de los problemas de desarrollo y proporcionar cambios a las conductas problemáticas del niño lo cual genera una mejor calidad de vida para todos los miembros de la familia.

Se considera que los padres de niños con TEA suelen ser los encargados de llevar a cabo protocolos basados en el comportamiento para mejorar la adquisición de habilidades y controlar el comportamiento problemático de sus hijos. En los estudios de 
Moore y Symons (2009), sobre la adherencia al tratamiento de padres de niños con TEA, los autores afirman que ese tema no ha sido investigado directamente, es decir, su investigación trata sobre la implementación de los tratamientos recomendados para manejar el comportamiento problemático de sus hijos que viven en casa. Los resultados encontrados fueron que la adherencia a las recomendaciones de tratamiento médico era significativamente mayor que la adherencia a las recomendaciones al tratamiento conductual $(p<.002)$. De las recomendaciones del tratamiento conductual, los padres informaron una mayor adherencia al refuerzo (81.7\%) que al castigo (68.9\%). Con relación al niño diagnosticado $(p<.002)$ y la interacción entre el diagnóstico por estado civil $(p<.05)$ se asoció significativamente con la adherencia referido al comportamiento y tratamiento médico, respectivamente. Otro hallazgo importante identificado son dos predictores significativos de adherencia: el diagnóstico predijo adherencia al tratamiento conductual (el síndrome de Asperger se asoció con niveles más bajos de adherencia en comparación con el diagnóstico de autismo o TEA) y la interacción entre el diagnóstico y el estado civil predijo adherencia al tratamiento médico.

Frente al modelo de intervención experto se ha desarrollado el modelo de intervención centrado en la persona o en nuestro caso, en la familia. En el Tratamiento Centrada en la Persona (TCP) los objetivos terapéuticos no están marcados por el profesional "experto", si no que se negocian o se establecen por parte del cliente (paciente). Una buena alianza terapéutica, independientemente del tipo de psicoterapia, es un elemento básico para conseguir una mejor adherencia. Se trata de una visión más integradora y sistémica, que parece permitir una mayor participación por parte del paciente, al considerar la relación entre "terapeuta-individuo-trastorno-entornocontexto sanitario" (Figura 2) como un todo interrelacionado, siendo el eje de la evaluación e intervención. En esta relación, el papel del terapeuta sería de permitir y facilitar el acercamiento del paciente que asume una posición activa, igualitaria y decisiva a la hora de acercarse, seguir, mantener o suspender libremente el plan de tratamiento (Miragaia, Artiles, y Fleta, 2009), o sea, implica al paciente para llevar a cabo un conjunto de comportamientos en la ausencia de supervisión por parte de un médico o clínico siendo esta medida un determinante críticamente importante para saber si ese tratamiento será efectivo (Moore y Symons, 2009).

Basado en este modelo, nuestro cuestionario trata de medir los elementos relacionados al individuo, a la familia, al niño, al social y el tratamiento que combinan entre si una serie de aspectos relacionales que conllevan al proceso de adherencia. 
Figura 2. Elementos integradores y sistemáticos de la adherencia

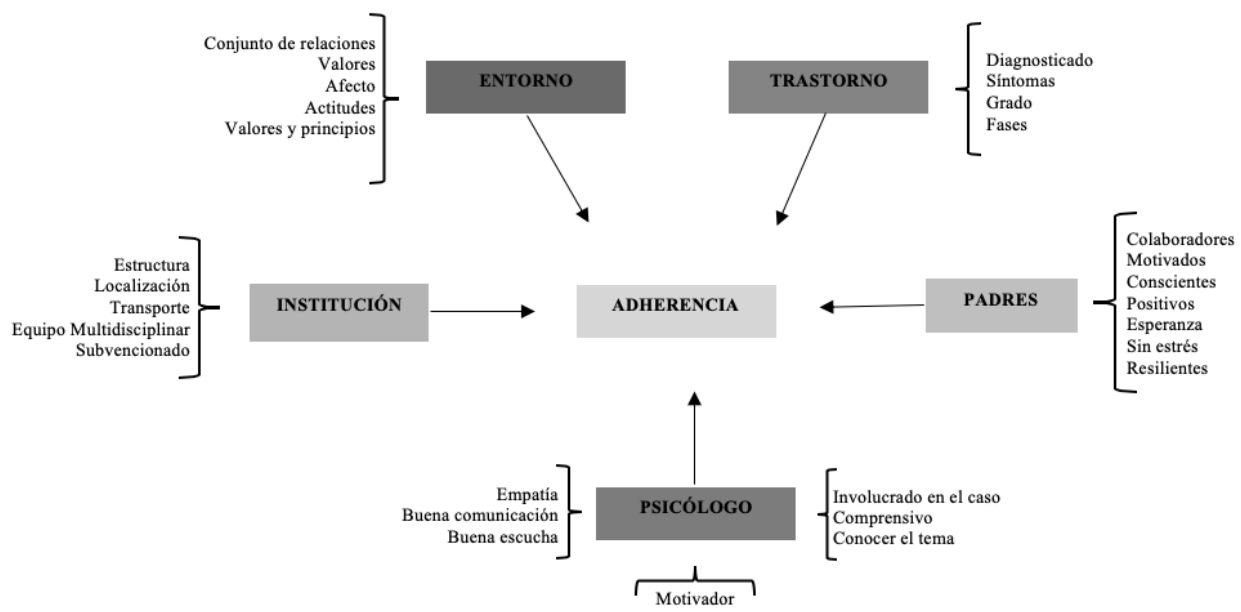

Elaboración propia a partir del marco teórico de Miragaia et al. (2009)

\section{MÉTODO}

Cuestionario ad-hoc inicialmente basado en 8 ítems de los que después se confirman en una versión más depurada 6, todo ello presentado originalmente en este trabajo, escala Likert de 4 puntos desde Nunca a Siempre. En el apartado de resultados se presentarán datos de fiabilidad y evidencias de validez de esta nueva escala. epidemiología de la enfermedad.

\section{Participantes}

El tamaño de la muestra fue de 128 participantes, recabados entre enero y mayo de 2013 en Valencia, España. En un primer momento de nuestra recogida, nos centramos en padres de niños entre 1 y 4 años de centros de atención temprana o específicos de TEA. En un segundo momento, fue ampliado el foco a padres de niños en centros escolares con aulas CyL (Comunicación y Lenguaje) hasta los 9 años, con el fin de obtener más evidencia de cara a la validación de la medida bajo estudio en varias situaciones.

En el estudio los participantes fueron divididos en: $47 \mathrm{CyL}, 43$ No CyL y 38 de CUDAP. De los 128 participantes, la encuesta fue aplicada a padres, madres y tutores, todos ellos fueron invitados a responder, una persona por unidad familiar y menor afectado. Además, por técnicas cualitativas (entrevista semiestructurada) se consideró la información de varios especialistas. 


\section{RESULTADOS}

El alfa con los 8 elementos de la escala es de .775 , es sabido que cuanto mayor sea el valor de alfa, mayor será la fiabilidad, y si se eliminan los dos ítems problemáticos anteriormente comentados (el 1 y el 8) apenas varía ese valor a pesar de reducir a tan sólo 6 ítems la escala, puesto que quedan en .767. En cualquiera de los casos es mayor que .7, el criterio normalmente exigido.

En los resultados de estadísticos de los 8 elementos de la escala, podemos observar la media, el índice de dificultad de las preguntas de la encuesta sobre la adherencia. Por ejemplo, la pregunta 2: “¿Realiza las actividades indicadas con la frecuencia adecuada?” con una puntuación de 3.133 y la pregunta 6: “Entiende las pautas que establece el profesional en el tratamiento de su hijo/a?" con 3.581 puntos. Significa que en un rango de valores de 1 a 4 (la escala Likert recordemos que se puntuaba desde Nunca a Siempre), un 3.6 aproximadamente estaría ubicado en los valores altos de la escala, es decir la muestra que responde en promedio tendría valor alto porque un 3.6 está muy próximo al rango 4 de la escala. Significa que la mayoría de los padres contestaron a menudo y es un valor muy alto.

Tabla 1. Listado de ítems propuestos

\begin{tabular}{lcccccccc}
\hline & \multicolumn{1}{c}{ Media } & \multicolumn{3}{c}{ Mediana } & Desv. Típ. Asimetría Curtosis & Mínimo Máximo \\
\hline ADHERENCIA 1 inv & 3.2609 & 3.0000 & .56331 & -.317 & 1.265 & 1.00 & 4.00 \\
ADHERENCIA 2 & 3.14 & 3.00 & .647 & -.535 & .997 & 1 & 4 \\
ADHERENCIA 3 inv & 3.5135 & 4.0000 & .56993 & -.654 & -.572 & 2.00 & 4.00 \\
ADHERENCIA 4 inv & 3.3805 & 3.0000 & .64529 & -.557 & -.628 & 2.00 & 4.00 \\
ADHERENCIA 5 & 3.47 & 4.00 & .644 & -.816 & .971 & 1 & 5 \\
ADHERENCIA 6 & 3.56 & 4.00 & .583 & -1.214 & 2.011 & 1 & 4 \\
ADHERENCIA 7 & 3.54 & 4.00 & .553 & -.643 & -.672 & 2 & 4 \\
ADHERENCIA 8 inv & 3.4679 & 3.0000 & .55392 & -.371 & -.928 & 2.00 & 4.00 \\
\hline
\end{tabular}

Analizando un segundo caso, es decir la escala reducida a 6 elementos, teniendo como criterio un número de 106 casos válidos y 22 de casos excluidos, es posible observar que el resultado presentado en el número Estadístico de Fiabilidad, el Alfa de Cronbach es exactamente de 0.767 como ya descrito arriba. Por consecuencia, podemos notar una pequeña diferencia en los resultados.

Analizando la fiabilidad de la escala de adherencia exclusivamente en la muestra de centros de AT podemos ver el resultado de Alfa de Cronbach de 0.811 para la adherencia con la medida definitiva de 6 elementos. Eso significa que, la escala tiene un grado de fiabilidad más satisfactorio en este colectivo que en el conjunto de la muestra total. 


\section{Resultados estructura factorial de la escala de adherencia}

Con el análisis factorial asumimos que las respuestas a los ítems son efectos de variables latentes, conceptos psicológicos no observables. El análisis factorial nos permite resumir la información de los datos en factores y facilitar la interpretación de las pruebas subsiguientes, estos análisis hacen nuestro trabajo como psicólogos aplicados más viable, pues sería imposible trabajar al nivel particular ítem a ítem y a la vez considerar en un mismo plano tantas variables, tanta información.

Para el estudio de la estructura de la escala de adherencia se propone una sola dimensión general o factor en que saturarían con signo positivo, en el sentido de la escala, la totalidad de ítems. Por tanto, previamente al análisis se ha invertido las puntuaciones de los ítems inversos o contrarios al rasgo. Cuando esta estructura se pone a prueba en los datos con técnicas de análisis factorial confirmatorio, observamos que sí ajusta al citado modelo unifactorial. Por tratarse de estimación robusta estos son los criterios en los que nos basamos para valorar el ajuste global: $(20)=27.036=p=.134$ (CFI=.966; IFI=.968), RMSEA=.064 con intervalo de confianza al 90\% (.000, .120).

Como se observa en la figura 3 , los ítems que más cargan o definen la adherencia son aquellos que expresan contenidos sobre la asimilación de pautas para trabajarlas en casa (ver Tabla 1) por orden los ítems 6 (si entienden las pautas), 7 (están de acuerdo con esas pautas) y 5 referidos a si conocen claramente las pautas de actuación en domicilio. Y los que menos, los ítems 1 y 8 , al borde del criterio recomendado para su exclusión, próximos en saturación al punto de corte de .3. Ambos ítems serán excluidos, quedando una escala breve de 6 ítems. En especial se elimina el ítem 8 por esta razón y por información cualitativa recabada de padres y profesionales se decide eliminar de la escala. El contenido del 8 referido a que los padres añadan sus propias pautas sin consultar no tiene por qué ser un indicador necesariamente de disfunción o mal funcionamiento. Puede comunicarlas porque las visitas no sean tan frecuentes, o en el extremo podría mostrar interés, dedicación.

Figura 3. Estimación coeficientes en AFC escala de adherencia por métodos robustos

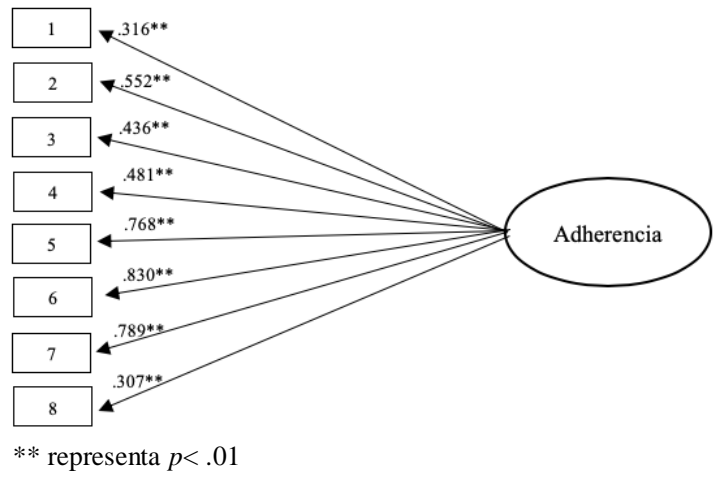


Tabla 2. Comportamiento de los ítems de la escala de adherencia

\begin{tabular}{|c|c|c|c|c|}
\hline & Nunca & $\begin{array}{l}\text { Pocas } \\
\text { veces }\end{array}$ & $\begin{array}{c}\mathrm{A} \\
\text { menudo }\end{array}$ & Siempre \\
\hline $\begin{array}{l}\text { 1. ¿Se olvida de hacer las actividades indicadas por los } \\
\text { profesionales del centro? }\end{array}$ & 1 & 2 & 3 & 4 \\
\hline $\begin{array}{l}\text { 2. ¿Realiza las actividades indicadas con la frecuencia } \\
\text { adecuada? }\end{array}$ & 1 & 2 & 3 & 4 \\
\hline $\begin{array}{l}\text { 3. Cuando observa avances en el desarrollo de su hijo/a, } \\
\text { ¿deja de aplicar las pautas indicadas? }\end{array}$ & 1 & 2 & 3 & 4 \\
\hline $\begin{array}{l}\text { 4. Cuando no encuentra cooperación por parte de su hijo/a, } \\
\text { ¿deja de aplicar las pautas indicadas? }\end{array}$ & 1 & 2 & 3 & 4 \\
\hline $\begin{array}{l}\text { 5. ¿Conoce las pautas que ha establecido el profesional para } \\
\text { llevar a cabo en casa con su hijo/a? }\end{array}$ & 1 & 2 & 3 & 4 \\
\hline $\begin{array}{l}\text { 6. ¿Entiende las pautas que establece el profesional en el } \\
\text { tratamiento de su hijo/a? }\end{array}$ & 1 & 2 & 3 & 4 \\
\hline $\begin{array}{l}\text { 7. ¿Está de acuerdo con las pautas prescritas por el } \\
\text { profesional para aplicar en casa? }\end{array}$ & 1 & 2 & 3 & 4 \\
\hline $\begin{array}{l}\text { 8. ¿Ha hecho algún cambio por sí mismo en cualquiera de } \\
\text { las pautas de tratamiento de su hijo/a, sin consensuarlo con } \\
\text { el profesional? }\end{array}$ & 1 & 2 & 3 & 4 \\
\hline
\end{tabular}

\section{Evidencias para la validez.}

En este apartado tenemos el propósito de comprobar si existe alguna relación o asociación entre las diversas variables de interés, en especial con la adherencia y si están asociadas antes de continuar con un análisis más sofisticado de mutuas interdependencias.

Este tipo de análisis presupone que las variables son ordinales o continuas y que la distribución de estas variables se acerca a la distribución normal. El coeficiente de correlación se proporcionará en forma estandarizada, esto significa que su rango va de -1 $\mathrm{a}+1$.

A continuación, se presentan los resultados de correlaciones lineales de Pearson entre las variables y los factores incluidos en la investigación, persiguiendo el objetivo de conocer las relaciones dos a dos con la adherencia. Este conocimiento nos aportará más evidencias sobre la validez de la medida de la adherencia en un marco más inclusivo, atendiendo también a las sociodemográficas y variables diagnósticas y del entorno familiar ¿Qué aspectos se relacionan más fuerte con la adherencia? ¿Cuáles de ellos de forma positiva y cuáles de forma negativa? ¿Hay un patrón de correlaciones diferente para la muestra total y para las submuestras específicas como la de familias que acuden a centros de atención temprana y los denominados de comunicación y lenguaje?

La tabla 3 muestra los coeficientes de relación entre la adherencia y 5 variables (esperanza, satisfacción con la vida, autonomía, cohesión y apoyo social) consideradas positivas y facilitadoras al proceso de adherencia al tratamiento. 


\begin{tabular}{|c|c|c|c|c|c|c|c|}
\hline & & 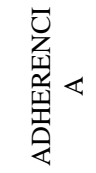 & 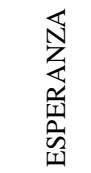 & 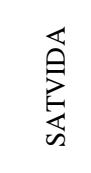 & 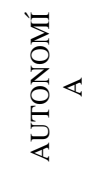 & 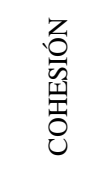 & 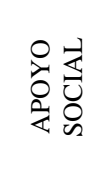 \\
\hline \multirow{3}{*}{ ADHERENCIA } & Correlación de Pearson & 1 & .127 & $.410 * *$ & .062 & $.245^{* *}$ & $.254 * *$ \\
\hline & Sig. (bilaeral) & & .178 & .000 & .512 & .009 & .000 \\
\hline & $\mathrm{N}$ & 115 & 114 & 113 & 115 & 113 & 115 \\
\hline \multirow{3}{*}{ ESPERANZA } & Correlación de Pearson & .127 & 1 & $.290 * *$ & .123 & -.006 & $.191 *$ \\
\hline & Sig. (bilaeral) & .178 & & .001 & .173 & .951 & .032 \\
\hline & $\mathrm{N}$ & 114 & 126 & 125 & 124 & 123 & 125 \\
\hline \multirow{3}{*}{ SATVIDA } & Correlación de Pearson & $.410 * *$ & $.290 * *$ & 1 & $.235^{* *}$ & $.452 * *$ & $.522 * *$ \\
\hline & Sig. (bilaeral) & .000 & .001 & & .009 & .000 & .000 \\
\hline & $\mathrm{N}$ & 113 & 125 & 125 & 123 & 122 & 124 \\
\hline \multirow{3}{*}{ AUTONOMÍA } & Correlación de Pearson & .062 & .123 & $.235 * *$ & 1 & $.216^{* *}$ & $.203^{*}$ \\
\hline & Sig. (bilaeral) & .512 & .173 & .009 & & .016 & .023 \\
\hline & $\mathrm{N}$ & 115 & 124 & 123 & 125 & 123 & 125 \\
\hline \multirow{3}{*}{ COHESIÓN } & Correlación de Pearson & $.245 * *$ & -.006 & $.452 * *$ & $.216^{* *}$ & 1 & $.562 * *$ \\
\hline & Sig. (bilaeral) & .009 & .951 & .000 & .016 & & .000 \\
\hline & $\mathrm{N}$ & 113 & 123 & 122 & 123 & 124 & 124 \\
\hline \multirow{3}{*}{$\begin{array}{l}\text { APOYO } \\
\text { SOCIAL }\end{array}$} & Correlación de Pearson & $.254 * *$ & $.191 *$ & $.522 * *$ & $.203^{* *}$ & $.562 * *$ & 1 \\
\hline & Sig. (bilaeral) & .006 & .032 & .000 & .023 & .000 & \\
\hline & $\mathrm{N}$ & 115 & 125 & 124 & 125 & 124 & 127 \\
\hline
\end{tabular}

** La correlación es signific ativa al nivel 0.01 (bilateral)

* La correlación es significante al nivel 0.05 (bilateral).

En el apartado teórico detallamos las características o factores que benefician o influían con el proceso de adherencia al tratamiento. Entre esos factores, hay los individuales, familiares y sociales, que son factores cruciales que determinarán una relación sea ella positiva o negativa con la adherencia.

Para las hipótesis estadísticas establecidas en este estudio, de entre las 5 mencionadas, se destacan 3 variables con mayor grado de relación con la adherencia: satisfacción con la vida, cohesión y apoyo social. La relación entre adherencia con satisfacción con la vida es positiva $(.410 * *)$, lo mismo pasa con la cohesión familiar $\left(.245^{* *}\right)$ y el apoyo social $(.254 * *)$. De entre las 5 variables elegidas, la correlación más fuerte se da con la satisfacción con la vida, el apoyo social también tras una relación positiva, no tan alta, pero significativa. Entre adherencia y las demás variables, esperanza (.127) y autonomía (.062), no surgen relaciones muy significativas.

Entre las variables, notamos que hay una asociación positiva significativamente importante entre apoyo social y satisfacción con la vida $(.522 * *)$; y relaciones significativas más fuertes entre apoyo social y cohesión familiar $(.562 * *)$ y cohesión familiar y satisfacción con la vida $(.452 * *)$. 
Los análisis de asociación entre las variables con aspectos negativos, se pueden observar en la tabla 4. Se puede examinar que todas las asociaciones menos la relación con la variable adaptación, obtuvieron resultados significativos.

Tabla 4. Relación de la adherencia con adaptación y con las variables que suponen medidas de aspectos negativos, y se esperan por tanto relaciones de signo negativo con la adhere ncia

\begin{tabular}{|c|c|c|c|c|c|c|c|}
\hline & & 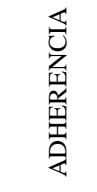 & 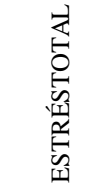 & 号 & 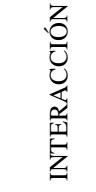 & 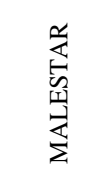 & 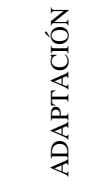 \\
\hline \multirow{3}{*}{ ADHERENCIA } & Correlación de Pearson & 1 & $-.326 * *$ & $-.215^{*}$ & $-.278 * *$ & $-.335 * *$ & .168 \\
\hline & Sig. (bilaeral) & & .000 & .023 & .003 & .000 & .079 \\
\hline & $\mathrm{N}$ & 115 & 113 & 112 & 111 & 113 & 113 \\
\hline \multirow{3}{*}{ ESTRÉSTOTAL } & Correlación de Pearson & $-.326 * *$ & 1 & $.764 * *$ & $.830 * *$ & $.870 * *$ & $-.257 * *$ \\
\hline & Sig. (bilaeral) & .000 & & .000 & .000 & .000 & .004 \\
\hline & $\mathrm{N}$ & 113 & 124 & 123 & 122 & 124 & 121 \\
\hline \multirow{3}{*}{ NIÑO } & Correlación de Pearson & $-.215^{*}$ & $.764 * *$ & & $.482 * *$ & $.479 * *$ & $-.249 * *$ \\
\hline & Sig. (bilaeral) & .023 & .000 & 1 & .000 & .000 & .006 \\
\hline & $\mathrm{N}$ & 112 & 123 & 123 & 122 & 123 & 120 \\
\hline \multirow{3}{*}{ INTERACCIÓN } & Correlación de Pearson & $-.278 * *$ & $.830 * *$ & $.482 * *$ & 1 & $.571 * *$ & -.075 \\
\hline & Sig. (bilaeral) & .003 & .000 & .000 & & .000 & .417 \\
\hline & $\mathrm{N}$ & 111 & 122 & 122 & 122 & 122 & 119 \\
\hline \multirow{3}{*}{ MALESTAR } & Correlación de Pearson & $-.335 * *$ & $.870 * *$ & $.479 * *$ & $.571^{* *}$ & 1 & $-.305 * *$ \\
\hline & Sig. (bilaeral) & .000 & .000 & .000 & .000 & & .001 \\
\hline & $\mathrm{N}$ & 113 & 124 & 123 & 122 & 124 & 121 \\
\hline \multirow{3}{*}{ ADAPTACIÓN } & Correlación de Pearson & .166 & $-.257 * *$ & $.249 * *$ & -.075 & $-.305 * *$ & 1 \\
\hline & Sig. (bilaeral) & .079 & .004 & .006 & .417 & .001 & \\
\hline & $\mathrm{N}$ & 113 & 121 & 120 & 119 & 121 & 124 \\
\hline
\end{tabular}

** La correlación es significativa al nivel 0.01 (bilateral).

* La correlación es significante al nivel 0.05 (bilateral).

Los resultados indican que cuanto mayor es el nivel de estrés hay menos adherencia $\left(-.326^{* *}\right)$, la relación entre el niño difícil (nivel de la escala de estrés parental) y la adherencia (-.215**), la interacción y la adherencia $\left(-.278^{* *}\right)$ y el malestar y la adherencia $\left(-.335^{* *}\right)$. Así pues, podemos considerar que los predictores negativos estrés y malestar parental son las variables que menos favorecen la adherencia.

\section{Modelo multivariado de explicación de antecedentes y consecuente de adherencia}

A la vista de la literatura y de los resultados basados en relaciones bivariadas mostrados en las páginas anteriores, a continuación, en la figura 4 se expresa el modelo de partida de esta tesis, con todas las variables implicadas en el estudio. Dado el número de casos en la muestra y el estudio bivariado (matrices de correlaciones entre las variables, se optó por un modelo más sencillo, prescindiendo de variables no explicativas 
en análisis preliminares anteriores (religiosidad, espiritualidad, autonomía, etc.) y en el que además se fijaron a cero las covarianzas entre las predictoras o exógenas del modelo a excepción de la conexión entre estrés parental y resiliencia que es fuerte y conocida.

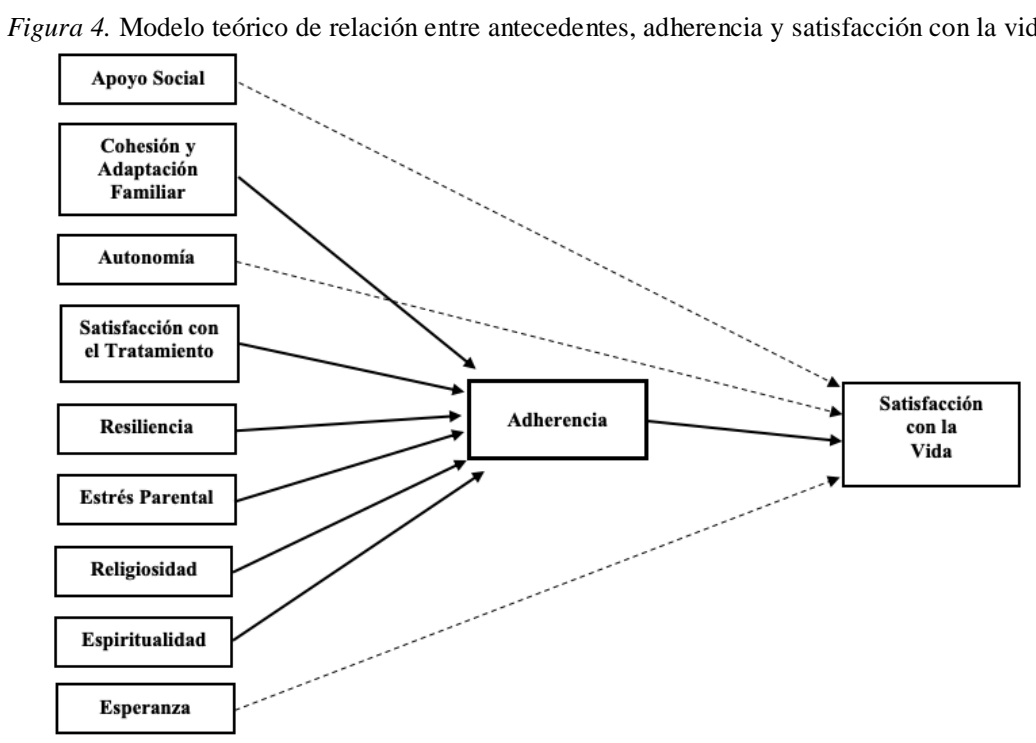

Cuando se puso a prueba, se estimó este modelo y constató que varias de las variables contempladas no tienen relación directa ni indirecta con el resto del modelo. Podemos ver que, de forma indirecta, pero no menos relacionadas, las variables actúan directamente o indirectamente, estas influencias no se pueden estudiar separadamente, pues este conjunto afectará la adherencia y esta a su vez a la satisfacción vital.

\section{DISCUSIÓN Y CONCLUSIONES}

Según nuestro planteamiento, la inclusión de la consideración de la adherencia de los cuidadores al tratamiento es un objetivo fundamental en programas dirigidos a la familia. Resulta claro que su participación en las sesiones, el saber manejar las situaciones inusitadas que puedan vivenciar en su día a día, traerá beneficios como mayor adaptación al TEA, menos estrés y una mejora continua en el desarrollo del niño. De este modo, la adherencia al tratamiento psicológico tiene como base la asistencia a las sesiones, el cumplimiento de las tareas, el conocimiento y el entendimiento del trastorno y la aceptación de las pautas, en común acuerdo, por parte de cuidadores y profesional socio-sanitario. 
Resulta, por tanto, apropiado identificar los elementos predictores para evaluar y mejorar la adherencia al tratamiento psicológico de los cuidadores que acuden a los centros de atención temprana con un hijo que tiene una sospecha o un diagnóstico confirmado de TEA, con el fin de progresar su adaptación a la situación a la que se enfrenta y mejorar la atención a su hijo. Dado que los cuidadores son los encargados de seguir el tratamiento, las intervenciones proporcionadas por los padres pueden mejorar la generalización de habilidades, la eficiencia del tratamiento y, sobre todo, pueden hacer que aumente el sentimiento de la autoeficacia de los padres (García et al., 2015).

La definición de un concepto general de adherencia aún sigue en proceso de construcción, pues, todavía no existe en la literatura un término universal o de consenso. Sin embargo, una idea compartida entre los autores consultados es que la adhesión al tratamiento desempeña un papel crítico en la determinación de la eficacia de las intervenciones (Hock, Kinsman, y Ortaglia, 2015). Un primer elemento del constructo es la asistencia al tratamiento, que se define simplemente como la presentación del paciente durante una sesión programada y acordada con el terapeuta. Un segundo aspecto es el compromiso de un paciente con el tratamiento, que puede ser definido mediante la adherencia al tratamiento o simplemente significa la aceptación de las estrategias de tratamiento (Gopalan et al., 2010), y más allá, hasta qué punto un paciente intenta implementar las estrategias aprendidas a través del tratamiento en ausencia de supervisión (Moore y Symons, 2009). Es cierto que la adherencia es un fenómeno complejo que está condicionado por múltiples factores, pero su esencia está relacionada al comportamiento humano unido principalmente por componentes subjetivos (Martín y Grau, 2004).

\section{REFERENCIAS}

Alcantud, F., y Alonso, Y. (2015). Trastornos del Espectro Autista. En E. (Ed.) Necesidades Educativas Especiales: Una mirada diferente (pp.7-26). Córdoba: Federación de Necesidades Educativas Especiales.

Fleta, J.L., Artiles, F.J., y Miragaia, J. (2009) Cuadernos de Psiquiatría Comunitaria: Adherencia Terapéutica. Revista de la Asociación Española de Neuropsiquiatría, 9(1), 13-28.

García, P., Posada, M., Martín, M., Santos, J., Bueno, G., y Canal, R. (2015). La detección e intervención tempranas en menores con trastorno del espectro autista. Siglo Cero, 46(2), 31-55. doi:10.14201/scero20154623155

Gopalan, G., Goldstein, L., Klingenstein, K., Sicher, C., Blake, C., y McKay, M.M. (2010). Engaging Families into Child Mental Health Treatmente: Updates and Special Considerations. Journal of the Canadian Academy of Child and Adolescent Psychiatry, $19,3$.

Guralnick, M.J. (2017). Early intervention for children with intellectual disabilities: An update. Journal of Applied Research in Intellectual Disabilities, 30(2), 211-229. doi:10.1111/jar.2017.30.issue-2 
Hock, R., Kinsman, A., y Ortaglia, A. (2015). Examining treatment adherence among parentsof children with autism spectrum disorder. Disability and Health Journal, 8(3), 407-413. doi:10.1016/j.dhjo.2014.10.005

Martín, L., y Grau, J. (2004). La investigación de la adherencia terapéutica como un problema de la psicología de la salud. Psicología y Salud, 14(1) 89-99.

McKay, M.M., y Bannon, W.M.J. (2004). Engaging families in child mental health services. Child and Adolescent Psychiatric Clinics of North America, 13(4), 905-921.

Miragaia, J., Artiles, F.J., y Fleta, J.L. (2009). Cuadernos de Psiquiatría Comunitaria: La adherencia en psicoterapia. Revista de la Asociación Española de Neuropsiquiatría. 9(1), 67-78.

Moore, T.R., y Symons, F.J. (2009). Adherence to behavioral and medical treatment recommendations by parents of children with autism spectrum. Journal of Autism and Developmental Disorders, 39(8), 1173-1184.

Nieto, L., y Pelayo, R. (2009). Revisión del concepto de adherencia al tratamiento y los factores asociados a ésta, como objeto de la psicología de la salud. Revista Académica e Institucional, 85, 61-75.

Organización Mundial de la Salud (2003). Adherence to Long-Term Therapies: Evidence for Action. Ginebra: OMS.

Recibido: 3 de junio de 2019

Recepción Modificaciones: 13 de junio de 2019

Aceptado: 2 de julio de 2019 